\title{
Equi-Biaxial Fatigue Behaviour of Magnetorheological Elastomers in Magnetic Fields
}

\author{
Yanfen Zhou \\ Technological University Dublin, yanfen.zhou@mydit.ie \\ Mark Johnson \\ Technological University Dublin, mark.johnson@tudublin.ie \\ Shipeng Wen \\ Beijing University of Chemical Technology
}

See next page for additional authors

Follow this and additional works at: https://arrow.tudublin.ie/cerart

Part of the Polymer and Organic Materials Commons

\section{Recommended Citation}

Zhou, Y., Johnson, M., Shipeng, W., Betts, A. and Jerrams, S. (2016) Equi-biaxial fatigue behaviour of magnetorheological elastomers in magnetic fields. Journal of Intelligent Material Systems and Structures, Published online before print July 5, 2016, Sage. doi:10.1177/1045389X16657204

This Article is brought to you for free and open access by the Centre for Elastomer Research at ARROW@TU Dublin. It has been accepted for inclusion in Articles by an authorized administrator of ARROW@TU Dublin. For more information, please contact arrow.admin@tudublin.ie, aisling.coyne@tudublin.ie,gerard.connolly@tudublin.ie. Funder: Irish Research Council

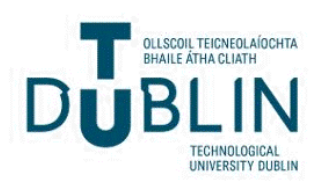




\section{Authors}

Yanfen Zhou, Mark Johnson, Shipeng Wen, Tony Betts, and Stephen Jerrams

This article is available at ARROW@TU Dublin: https://arrow.tudublin.ie/cerart/13 


\title{
Equi-biaxial fatigue behaviour of magnetorheological elastomers in magnetic fields
}

Yanfen Zhou ${ }^{1 \mathrm{a}}$, Mark Johnson ${ }^{\mathrm{a}}$, Shipeng Wen ${ }^{\mathrm{b}}$, Anthony Betts ${ }^{\mathrm{c}}$, Stephen Jerrams ${ }^{\mathrm{a}}$

${ }^{a}$ Centre for Elastomer Research, Focas Research Institute, Dublin Institute of Technology, Kevin Street, Dublin 8, Ireland

${ }^{\mathrm{b}}$ Centre of Advanced Elastomer Materials, Beijing University of Chemistry and Technology, PR China

${ }^{\mathrm{c}}$ Applied Electrochemistry Group, Focas Research Institute, Dublin Institute of Technology, Kevin Street, Dublin 8, Ireland

\begin{abstract}
The equi-biaxial fatigue behaviour of silicone based magnetorheological elastomers (MREs) in external magnetic fields was studied. Wöhler curves relating fatigue life to stress amplitude and dynamic stored energy for MREs with a range of magnetic particle contents were derived. It was found that the fatigue life of MREs in magnetic fields was higher than that without magnetic fields. Under constant stress amplitude conditions, the presence of magnetic fields resulted in longer times for the samples to undergo large deformations and thus complex modulus $\left(\mathrm{E}^{*}\right)$ decreased at a slower rate during the fatigue process, especially for low stress amplitudes. MRE samples tested in the presence of magnetic fields reached limiting values of $\mathrm{E}^{*}$ at failure ranging from 1.28 $\mathrm{MPa}$ to $1.44 \mathrm{MPa}$. The application of magnetic fields was found to have negligible influence on the damping loss factor of MREs containing various volume fractions of carbonyl iron particles.
\end{abstract}

Keywords: magnetorheological elastomers, equi-biaxial fatigue, magnetic field, fatigue life, complex modulus, dynamic stored energy

\section{Introduction}

Magnetorheological elastomers (MREs) are smart materials with rheological properties that can be controlled instantaneously and reversibly by the application of an external magnetic field (Schubert and Harrison, 2015). Structured (Zhang et al., 2010; Ju et al., 2015) and isotropic (Lokander and Stenberg, 2003; Gong et al., 2005) MREs can be fabricated depending on whether an external magnetic field is applied or not during the curing process. Due to the field dependent rheological response of MREs, they are interesting candidates for adaptive tuned vibration absorbers, stiffness tuneable mounts and suspensions and variable impedance surfaces (Watson, 1997; Elie et al., 1998; Fuchs et al., 2006; Hoang et al., 2009).

Magnetic fields have a significant influence on the properties of MREs (Lokander et al., 2004) and these properties are strongly dependent on the field strength. Changes in the properties of an MRE under an external magnetic field, generally described as the MR effect, are related to the magnetic particles tendency to change their positions under the influence of an applied magnetic field (Boczkowska and Awietjan, 2012). Interactions between particles in magnetic fields draw them closer, resulting in changes in the stress-strain behaviour of the MRE (Farshad and Le Roux, 2005). In most cases, a magnetic field induces an increase in the stiffness of the composite (Boczkowska et

\footnotetext{
${ }^{1}$ Corresponding author. Tel: +35301 4027919. Email: yanfen.zhou@mydit.ie
} 
al., 2007). In some studies, it was stated that the MR effect also includes a magnetically induced change in damping properties (Bellan and Bossis, 2002).

Fatigue is one of the most important characteristics of modern materials. Models for predicting the fatigue life of elastomers mainly follows two approaches: the crack nucleation method (Cadwell et al., 1940; Mars and Fatemi, 2002) and the crack growth method (Rivlin and Thomas, 1953). More recently, several sophisticated multi-axial fatigue life predictors, based on critical plane-based approaches, have been developed for rubber (Mars, 2002; Ayoub et al., 2012). MREs are intended to be used as structural materials in applications where the loading is usually dynamic (Boczkowska and Awietjan, 2009). This unquestionably leads to the investigation of the dynamic behaviour of MREs, particularly under multi-axial deformation. Previous research has concentrated on the equi-biaxial fatigue behaviour of isotropic and anisotropic silicone based MREs in the absence of external magnetic fields (Zhou et al., 2014; Zhou et al., 2015). The fatigue behaviour of MREs in the presence of magnetic fields is reported for the first time here. Specifically, research into the equi-biaxial fatigue behaviour of MREs in the presence of external magnetic fields was studied with the ultimate aim of providing parameters for the design of MRE based rubber components.

\section{Materials and Methods}

\subsection{Materials}

Isotropic silicone rubber based MREs were fabricated by incorporating different volume fractions $(15 \%, 20 \%, 25 \%, 30 \%$ and $35 \%)$ of soft carbonyl iron (CI) magnetic particles $(\mathrm{d} 50=6.0-7.0 \mu \mathrm{m}, \mathrm{CS}$ grade, BASF) into a two component room temperature vulcanized (RTV) silicone rubber. Firstly, silicone rubber was mixed with a catalyst at a 10:1 ratio, before carbonyl iron particles were introduced to the mixture which was mechanically stirred to distribute the particles evenly in the elastomer matrix. The whole mixture was degassed in a vacuum to remove entrapped air bubbles and then poured into a mould. After further degassing in the mould, the compound was kept at room temperature for 48 hours to allow solidification. The thickness of the fabricated samples was nominally $1 \mathrm{~mm}$.

\subsection{Methods}

Equi-biaxial fatigue tests were conducted on each sample by using the bubble inflation system similar to that decsribed in a previous paper (Zhou et al., 2014). A Halbach array was set up around the bubble inflation testing orifice as depicted schematically in Figure 1(a). The magnetic flux density distribution is shown in Figure 1(c) and (d). The position of the Halbach array ensured the specimens tested were subjected to a relatively uniform mean magnetic field strength of about $400 \mathrm{mT}$ during inflation and deflation. 
(a)

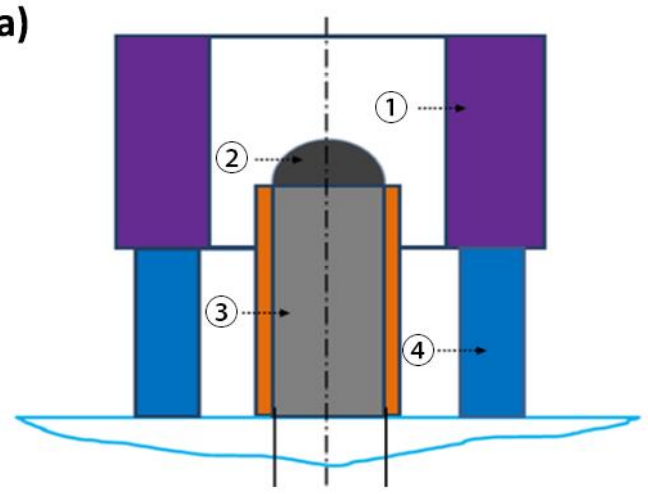

Equibiaxial test setup: (1) Halbach array;

(2) Inflated MRE sample; (3) Testing orfice;

(4) Supporting item

(c)

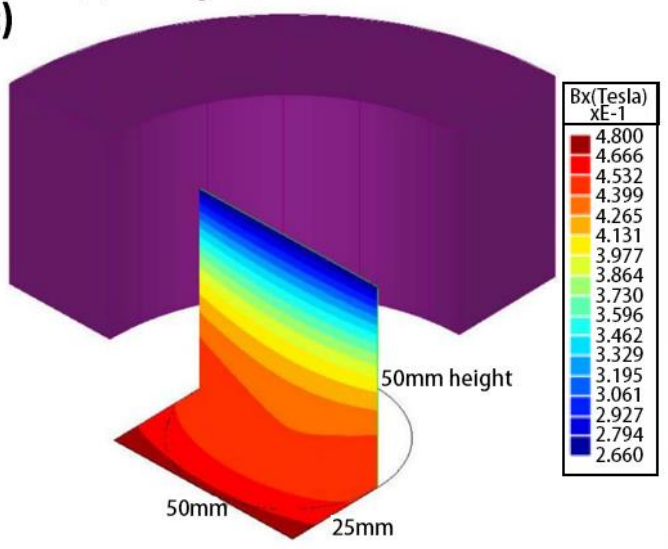

Variation of flux density in the vertical plane va at the array centre

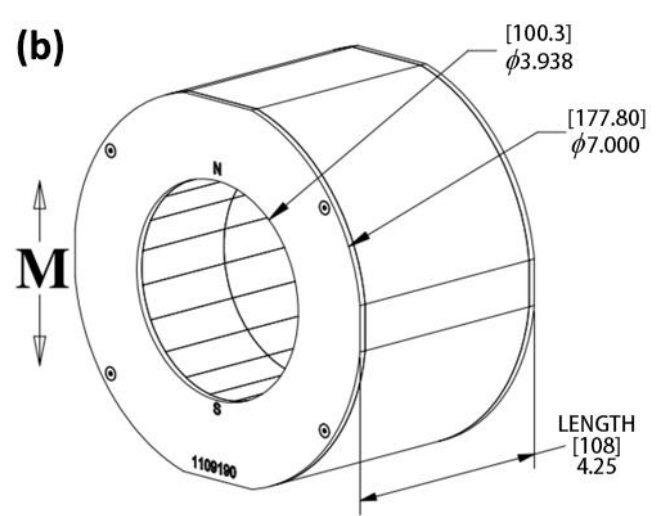

A diagrammatic view of the Halbach Array (dimensions in inches and $\mathrm{mm}[*]$ )

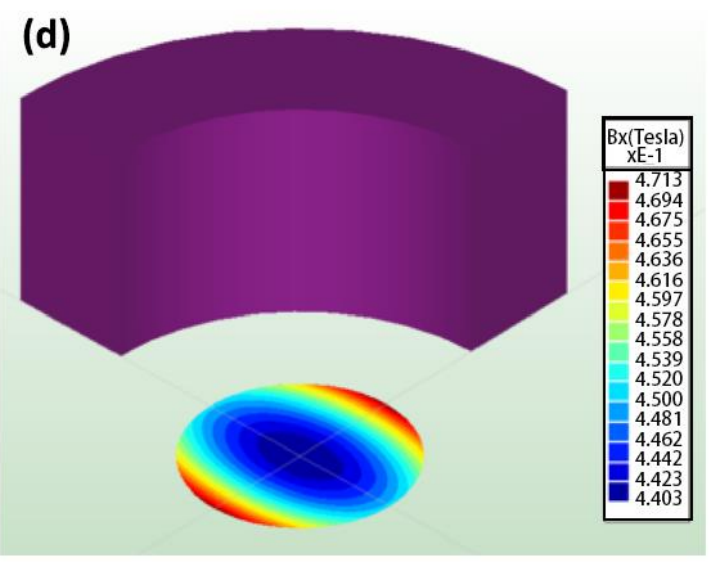

Figure 1 Equi-biaxial fatigue test setup and the magnetic field used

\section{Results and Discussion}

\subsection{Fatigue life}

Figure 2 shows the Wöhler curves of MREs containing different volume fractions of carbonyl iron particles tested in the absence and in the presence of the magnetic field provided by the Halbach array. Also, Table 1 shows the increases in fatigue life with the magnetic field applied for the specific alternating stress of $0.75 \mathrm{MPa}$. As can be seen, for MREs with the same CI content, the fatigue life was higher when the samples were tested in a magnetic field. The increases in fatigue life can be attributed to the enhanced interactions between the particles and between the particles and the matrices in the presence of magnetic fields. 
Table 1 Fatigue life of MREs with different CI contents tested with and without magnetic fields at a stress amplitude of $0.75 \mathrm{MPa}$

\begin{tabular}{ccc}
\hline \multirow{2}{*}{ CI volume content $(\boldsymbol{\%})$} & \multicolumn{2}{c}{$\boldsymbol{N}_{\boldsymbol{f}}(\mathbf{C y c l e s}$ to failure) } \\
\cline { 2 - 3 } & with magnetic field & without magnetic field \\
\hline $\mathbf{1 5}$ & 2756 & 2146 \\
$\mathbf{2 0}$ & 1945 & 1368 \\
$\mathbf{2 5}$ & 1758 & 1364 \\
$\mathbf{3 0}$ & 3055 & 1314 \\
$\mathbf{3 5}$ & 6982 & 4033 \\
\hline
\end{tabular}

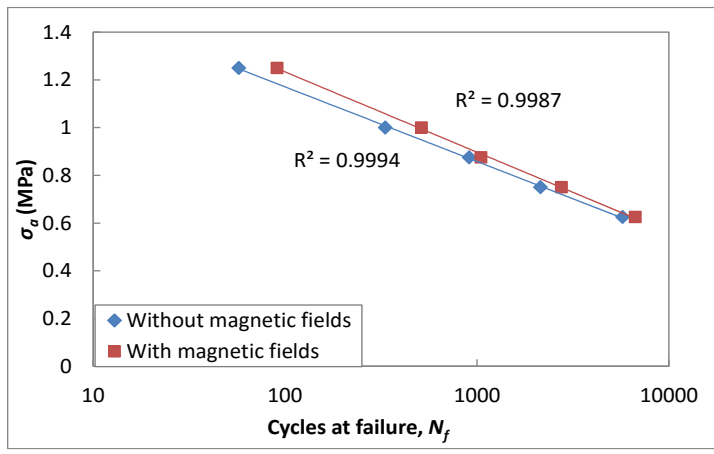

(a)

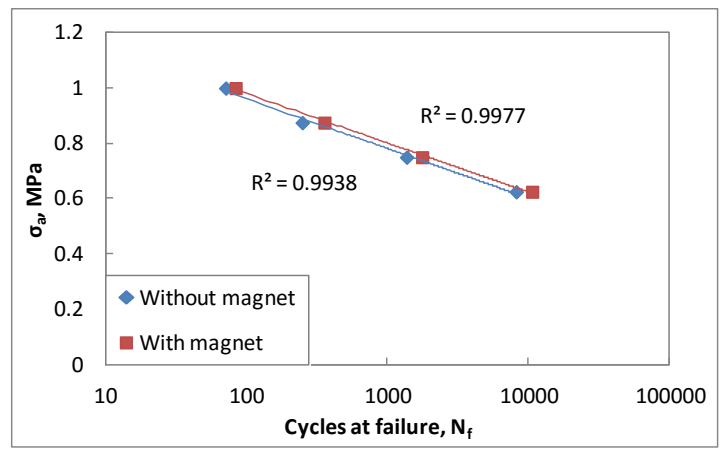

(c)

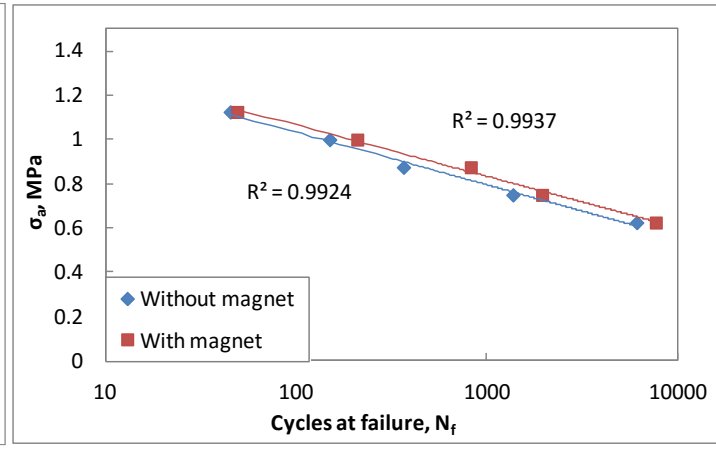

(b)

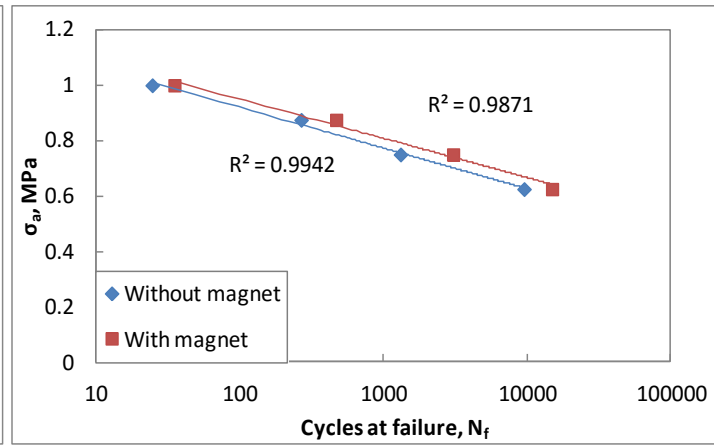

(d)

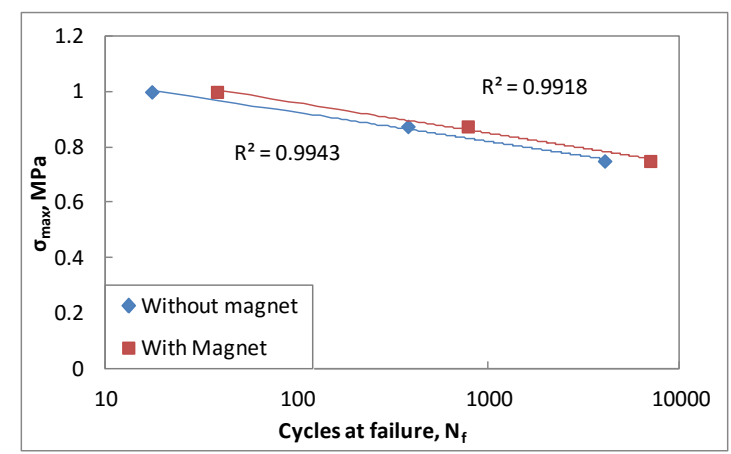

(e)

Figure 2 Wöhler curves of various MREs tested without and with a magnet, (a) 15\%; (b) $20 \%$; (c) $25 \%$; (d) $30 \%$ and (e) $35 \%$ 
Equations for fatigue life prediction (in magnetic fields), relating life $\left(N_{f}\right)$ to engineering stress amplitudes $\left(\sigma_{a}\right)$ for SR based MREs were derived as follows:

$\ln \left(N_{f}\right)=\left(\sigma_{a}-A_{2}\right) / A_{1}$

where $A_{1}$ and $A_{2}$ are material specific constants dependent on magnetic particle content. The values of $A_{1}$ and $A_{2}$ for MREs with various CI contents are listed in Table 2.

Table 2 Values for $A_{1}$ and $A_{2}$ for MREs tested in magnetic fields

\begin{tabular}{ccc}
\hline CI volume content $(\boldsymbol{\%})$ & $\boldsymbol{A}_{\boldsymbol{1}}\left(\mathbf{m m}^{\mathbf{2}} \mathbf{/ N}\right)$ & $\boldsymbol{A}_{2}$ (no units) \\
\hline 15 & -0.146 & 1.9061 \\
20 & -0.101 & 1.5285 \\
25 & -0.077 & 1.3352 \\
30 & -0.064 & 1.2560 \\
35 & -0.048 & 1.1785 \\
\hline
\end{tabular}

\subsection{Stress-strain behaviour}

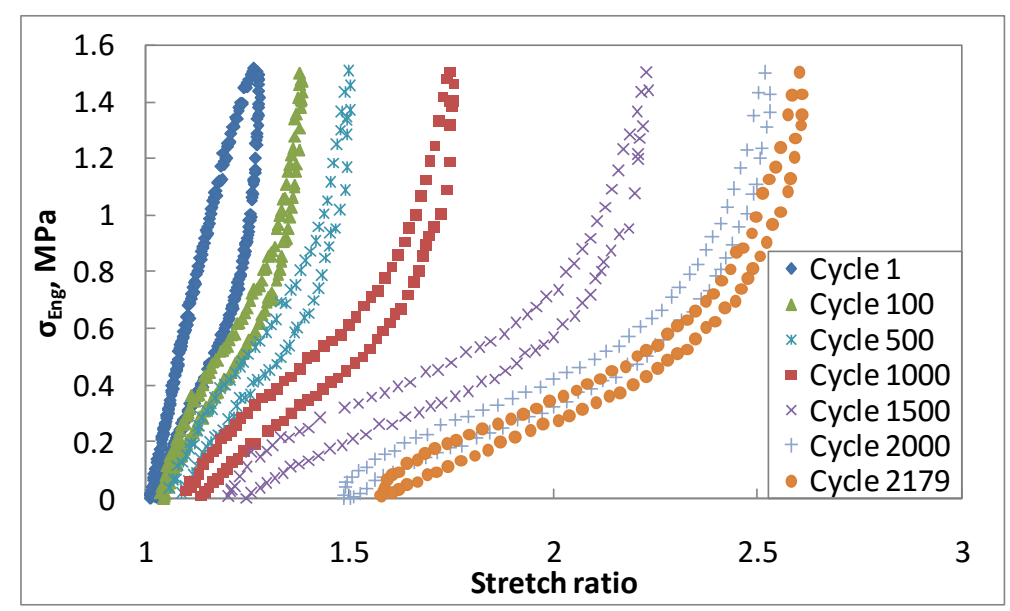

(a)

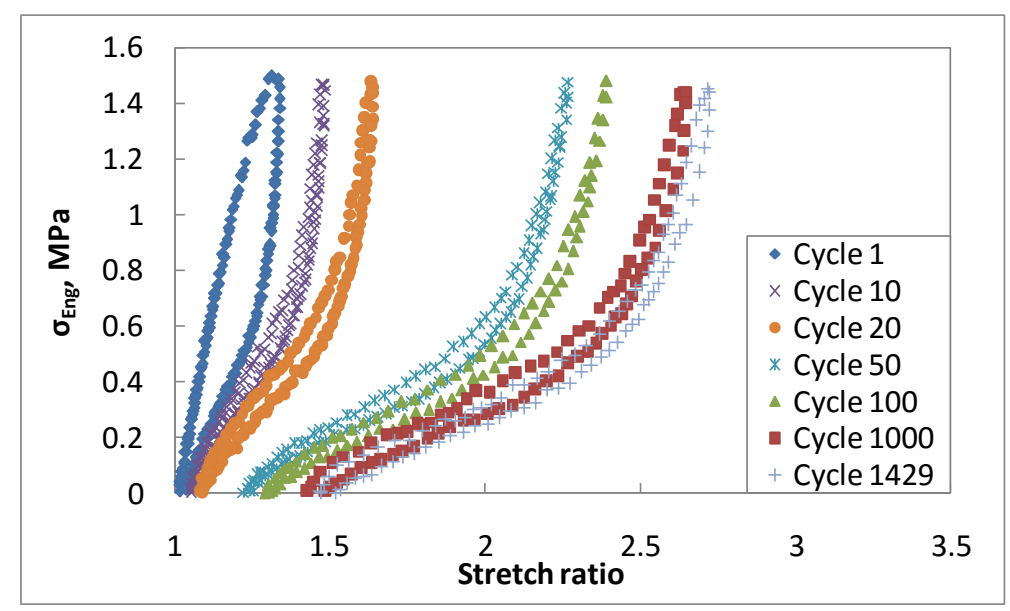

(b)

Figure 3 Stress-stretch ratio curves for MREs with $20 \% \mathrm{CI}$ during a fatigue test at $\sigma_{\mathrm{a}}=$ $0.75 \mathrm{MPa}$, (a) with and (b) without magnetic fields 
Figure 3 shows the stress-strain behaviour of MRE samples during a fatigue test in the presence and in the absence of magnetic fields. In this figure, the behaviour of the $20 \%$ CI content samples is depicted, but curves were similar for all CI contents. Stress softening and hysteresis continued as cycles accumulated in both cases. However, the stress-strain behaviour of MRE samples was greatly influenced by the application of external magnetic fields. As can be noted from Figure 3, at the same maximum engineering stress of 1.5 $\mathrm{MPa}$, the stretch ratio for the same cycle was significantly lower than when the sample was tested in a magnetic field (with the exception of the first loading), e.g. at 100 cycles, the maximum stretch ratio was 2.39 when the sample was tested without a magnetic field while it was only 1.38 in the presence of a magnetic field. It is known that an external magnetic field causes the interaction between particles to increase, hence their attractions to each other become greater (Böse and Röder, 2009). However, compared with when the field is absent, these enhanced interactions between magnetic particles restricted the mobility of the elastomer chains, leading to smaller deformations for the same number of cycles.

\section{$3.3 \operatorname{Modulus}\left(E^{*}\right)$}

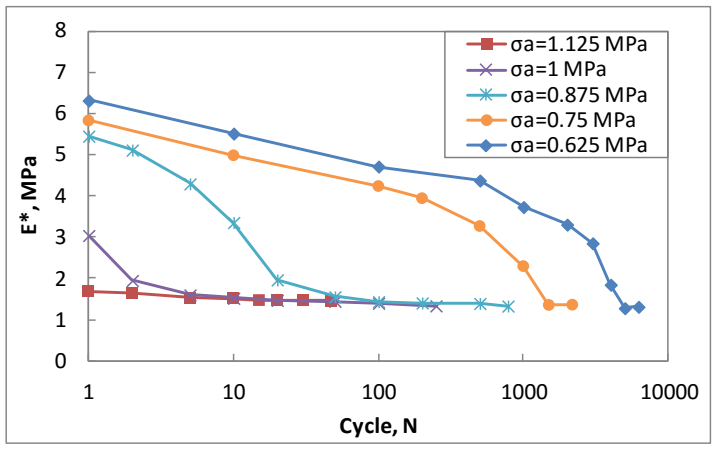

(a)

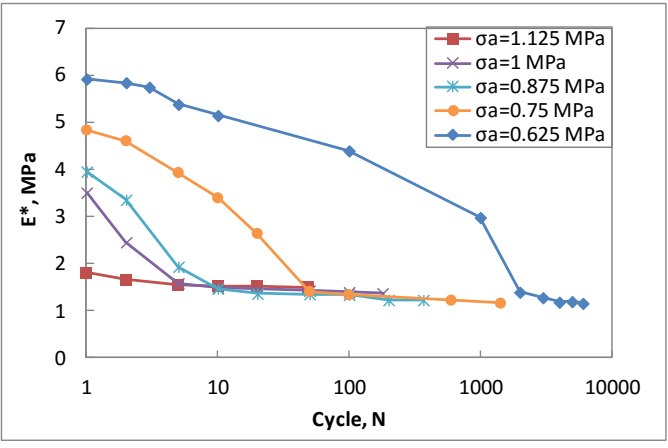

(b)

Figure 4 Evolution of $E^{*}$ for MREs with $20 \%$ CI at various stress amplitudes, (a) with and (b) without magnetic fields

Mechanical strength deterioration during the fatigue process was characterized by a diminution in complex modulus $\left(\mathrm{E}^{*}\right)$. Changes in $\mathrm{E}^{*}$ at various stress amplitudes for MRE samples tested with and without magnetic fields are shown in Figure 4. For both situations, $E^{*}$ decreased with accumulated cycles regardless of the stress levels applied. However, the decrease in $\mathrm{E}^{*}$ was more gradual when the samples were tested in magnetic fields and this was in greater evident at lower stress amplitudes. This result coincided with the stress-strain behaviour described in Section 3.2. Due to the enhanced interactions between the particles and between the particles and elastomeric matrices in the presence of magnetic fields, it took longer for specimens to undergo large deformations and for values of $\mathrm{E}^{*}$ to reach the failure limit at a similar engineering stress.

However, irrespective of whether the magnetic field was applied or not, MRE samples failed at a limiting level of $E^{*}$. Equi-biaxial fatigue tests of MRE samples with a range of magnetic particle contents in the presence of a magnetic field further strengthened 
this finding. As can be seen from Figure 5, for MREs with various volume fractions of carbonyl iron particles, $E^{*}$ decreased with the accumulation of cycles as a result of stress softening at each stress amplitude applied in the tests. Regardless of the stress amplitude applied, all samples failed at a limiting value of $\mathrm{E}^{*}$ ranging between 1.28 and 1.44 MPa (refer to Table 3). When compared with previous results without magnetic fields (Zhou et al., 2015), it was found that $E^{*}$ at failure for MREs with various particle content were slightly higher in the presence of magnetic fields, but this was in a relatively small range.

The instantaneous increase in modulus experienced by an MRE when a magnetic field is applied is well known and has been researched extensively. The research reported here suggests that fatigue life is also higher for components actuated in the presence of a magnetic field. Moreover, it was shown that the reduction in complex modulus $\left(\mathrm{E}^{*}\right)$ associated with dynamic loading was less pronounced when a magnetic field was applied. This has implications for the resilience of MRE based components, as Alshuth et al (Alshuth et al., 2002) have shown that $\mathrm{E}^{*}$ can be used as an indicator of fatigue failure and a design criterion for conventional rubber components. This finding was reinforced for equi-biaxial dynamic loading of elastomers by Murphy (Murphy, 2010).

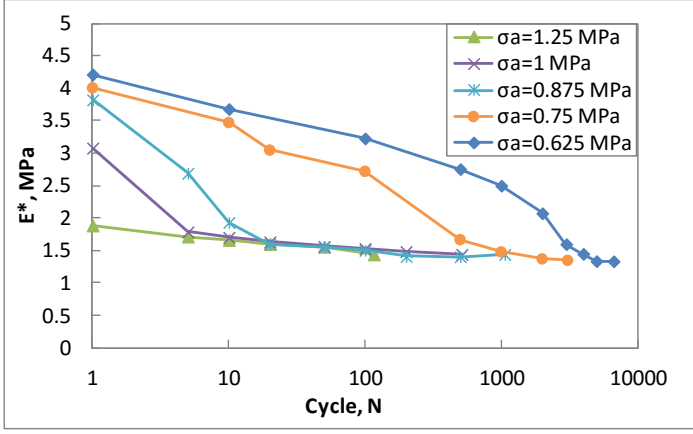

(a)

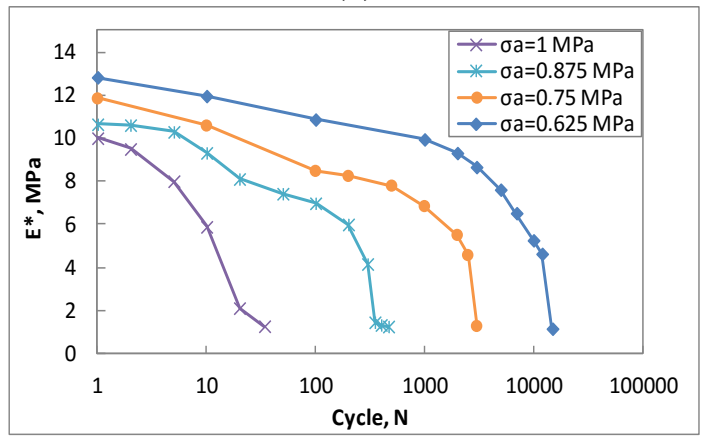

(c)

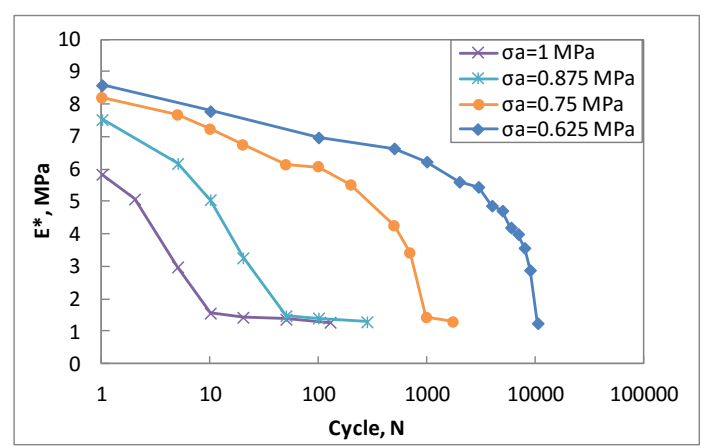

(b)

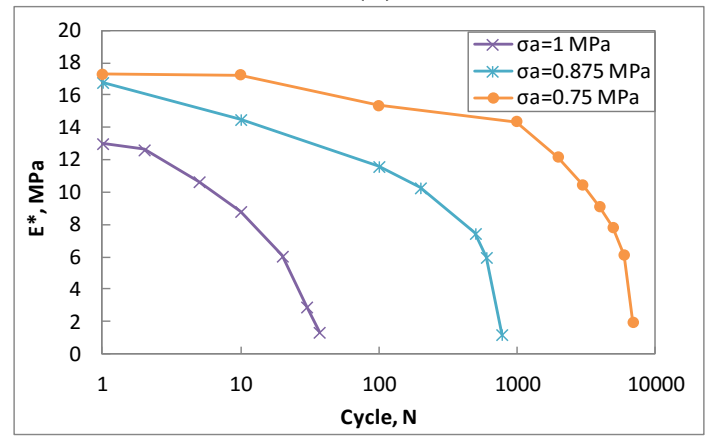

(d)

Figure 5 Evolution of $E^{*}$ during testing for MREs with various CI content (a) $15 \%$; (b) $25 \%$; (c) $30 \%$; (d) $35 \%$. Samples were tested in the presence of a magnetic field 
Table $3 \mathrm{E}^{*}$ at failure for MREs tested with magnetic fields

\begin{tabular}{cc}
\hline CI volume content $(\boldsymbol{\%})$ & $\mathbf{E}^{*}$ at failure (MPa) \\
\hline 15 & $1.44 \pm 5.58 \%$ \\
20 & $1.37 \pm 3.25 \%$ \\
25 & $1.33 \pm 6.12 \%$ \\
30 & $1.27 \pm 4.24 \%$ \\
35 & $1.28 \pm 5.99 \%$ \\
\hline
\end{tabular}

\subsection{Energy}

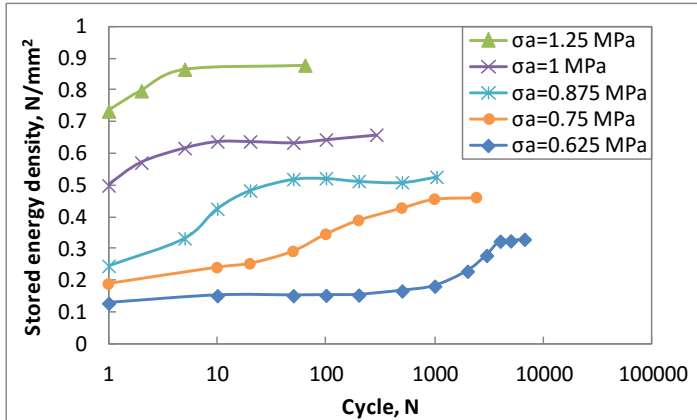

(a)

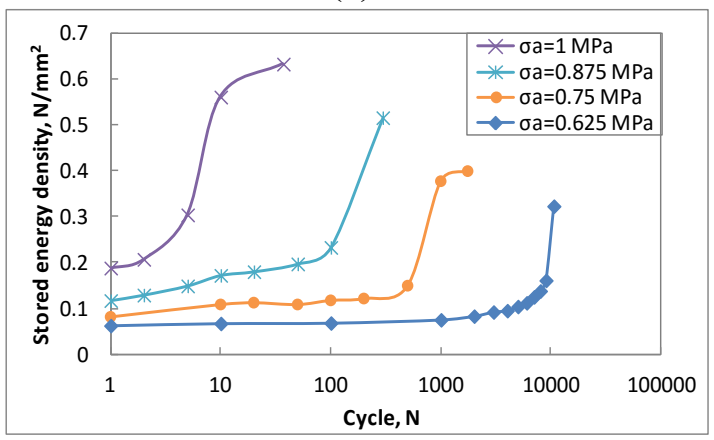

(c)

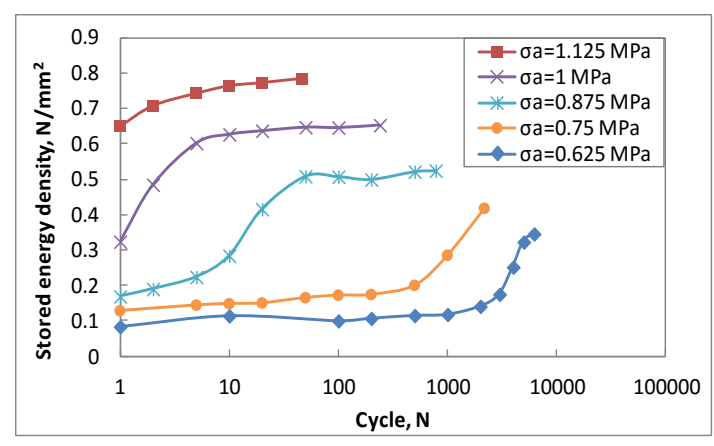

(b)

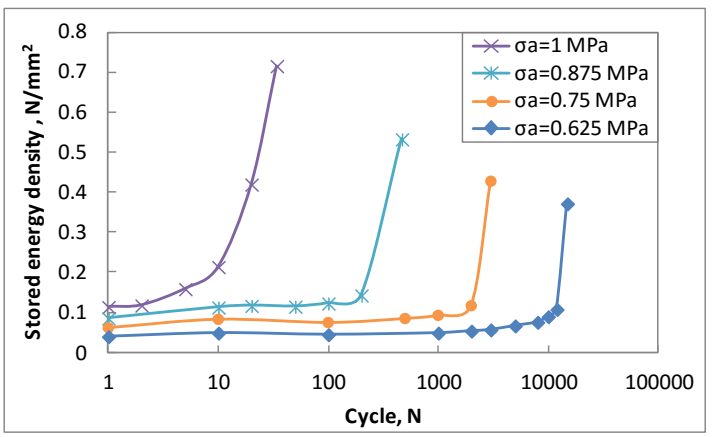

(d)

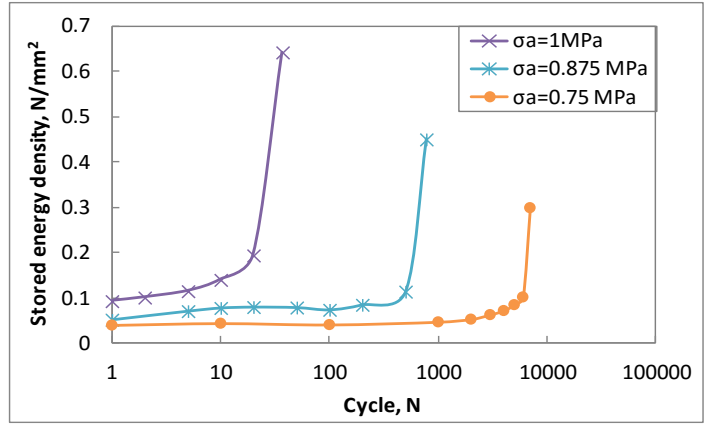

(e)

Figure 6 Evolution of stored energy density versus cycles for isotropic MREs with different CI content, (a) 15\%; (b) 20\%; (c) 25\%; (d) 30\%; (e) 35\%. Samples were tested in the presence of a magnetic field

In viscoelastic materials, some of the total deformation energy input is stored and recovered during each cycle whilst some is dissipated as heat. Dynamic stored energy (per unit volume), which is defined as the strain energy available in the unloading cycle, 
can be determined from stress-strain curves (Roylance, 2001). The evolution of dynamic stored energy against cycles for the tests on isotropic MREs with various CI contents is shown in Figure 6.

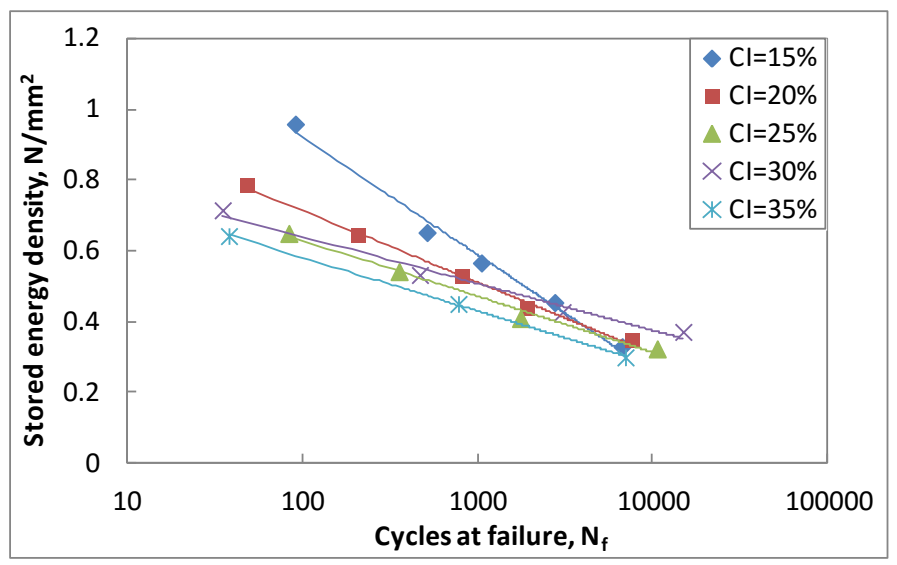

Figure 7 Stored energy density at failure versus cycles at failure for isotropic MREs with different CI content. Samples were tested in the presence of a magnetic field

When plotted against cycles to failure, the stored energy density at failure for a range of stress amplitudes was found to decrease linearly for each sample as shown in Figure 7. This indicates that the stored energy criterion can be used as a plausible fatigue life predictor for MREs subjected to external magnetic fields. A stored energy-based equation for fatigue life prediction can be written by relating life $\left(N_{f}\right)$ to stored energy density $\left.\left(W_{s}\right)\right)$ as represented by Eqn. 2.:

$\ln \left(N_{f}\right)=\left(W_{s}-B_{2}\right) / B_{1}$

where $B_{1}$ and $B_{2}$ are material specific constants dependent on the magnetic particle content. The values for $B_{1}$ and $B_{2}$ for MREs with various CI contents are given in Table 4.

Table 4 Values for $B_{1}$ and $B_{2}$ for MREs tested in magnetic fields

\begin{tabular}{ccc}
\hline CI volume content $(\boldsymbol{\%})$ & $\boldsymbol{B}_{\boldsymbol{1}}\left(\mathbf{m m}^{\mathbf{2}} / \mathbf{N}\right)$ & $\boldsymbol{B}_{\mathbf{2}}\left(\mathbf{m m}^{\mathbf{2}} \mathbf{N}\right)$ \\
\hline 15 & -0.144 & 1.584 \\
20 & -0.088 & 1.1174 \\
25 & -0.069 & 0.9448 \\
30 & -0.058 & 0.9049 \\
35 & -0.066 & 0.8827 \\
\hline
\end{tabular}

\subsection{Damping loss factor $(\eta)$}

Evaluating the damping capabilities of MREs is important in understanding their potential application in various damping and vibration isolation devices. To describe the damping properties of the material, parameters such as the damping loss factor are generally used (Yim et al., 2003). For isotropic MREs, tested with and without magnetic 
fields, the variations of damping loss factor with respect to cycles are shown in Figure 8 and Figure 9 respectively. It can be seen that dynamic loading induced an overall decrease in the damping loss factor throughout the entire fatigue process. However, for each material, $\eta$ decreased primarily in the first few cycles and reached a limiting value at failure irrespective of the stress amplitudes applied. This indicates that the damping properties of MREs can be maintained at a stable level during the entire service life after conditioning has taken place. The values of $\eta$ at failure for MREs with various CI contents are shown in Table 5. When $\eta$ was compared for MREs tested with and without magnetic fields, it was found that the presence of a magnetic field did not have significant influence on the damping loss factor during the entire fatigue process, although the value of $\eta$ at failure was generally slightly higher when a magnetic field was applied.

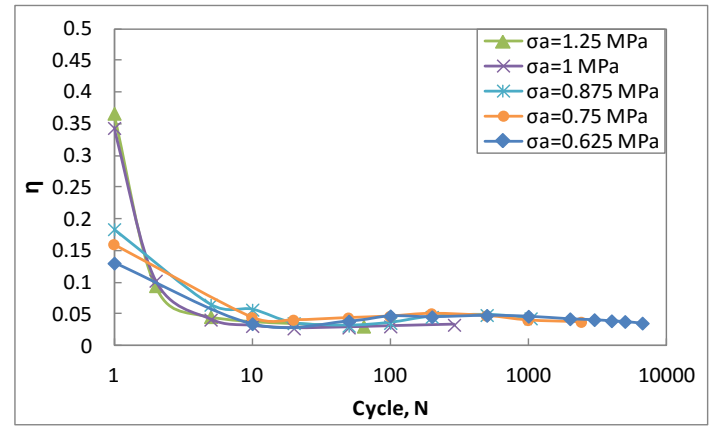

(a)

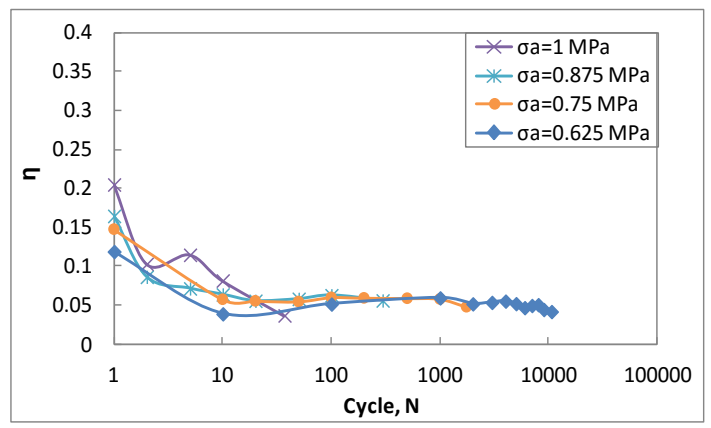

(c)

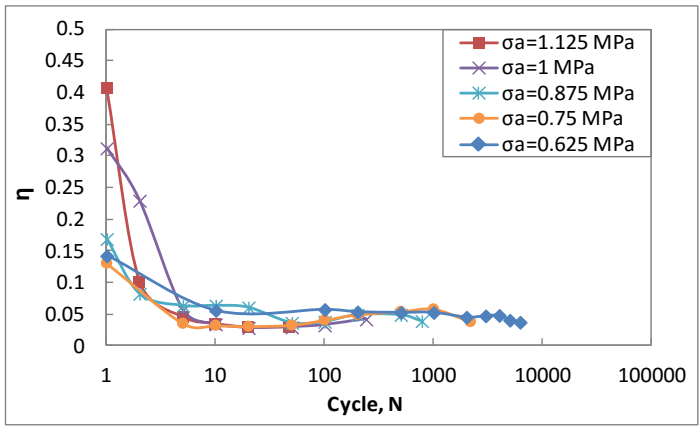

(b)

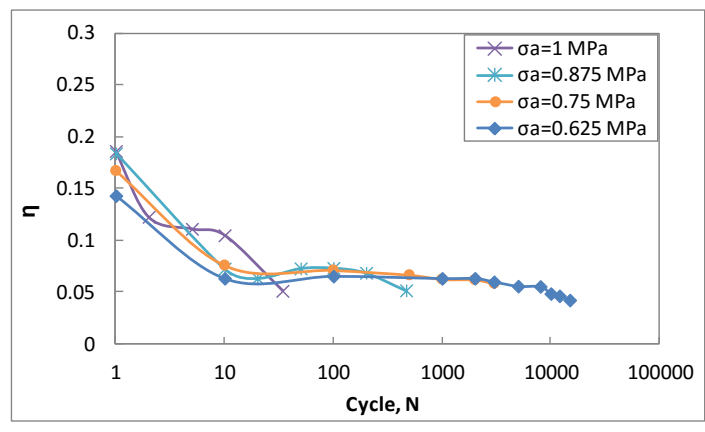

(d)

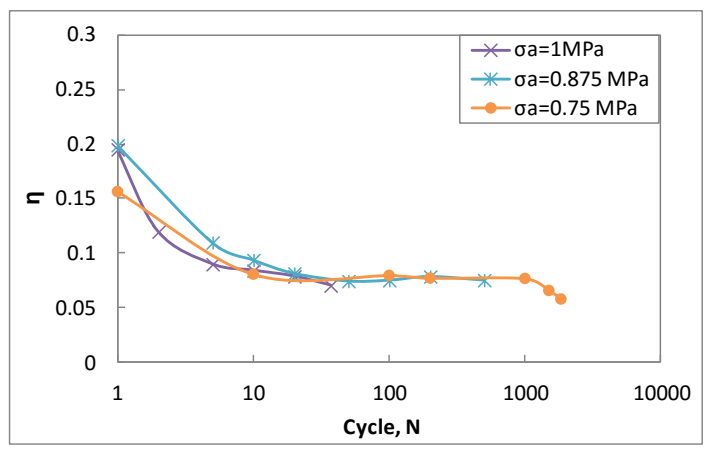

(e)

Figure 8 Damping loss factor versus cycles for isotropic MREs with different CI content, (a) $15 \%$; (b) $20 \%$; (c) $25 \%$; (d) $30 \%$; (e) $35 \%$. Samples were dynamically tested in the presence of a magnetic field 


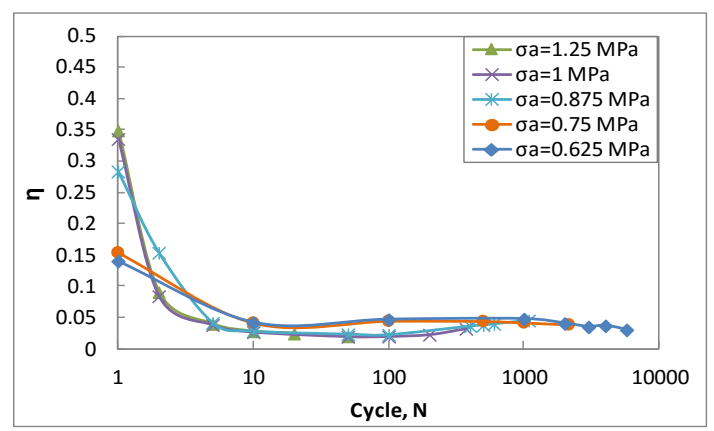

(a) $15 \%$

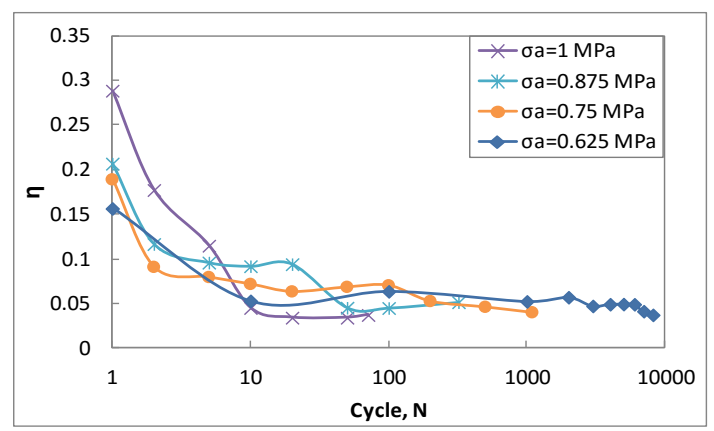

(c) $25 \%$

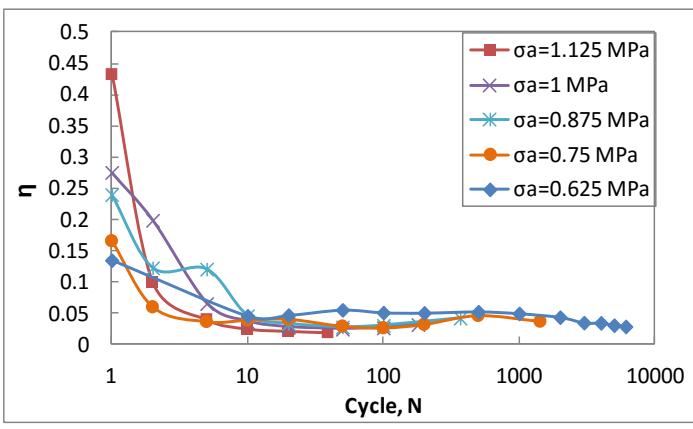

(b) $20 \%$

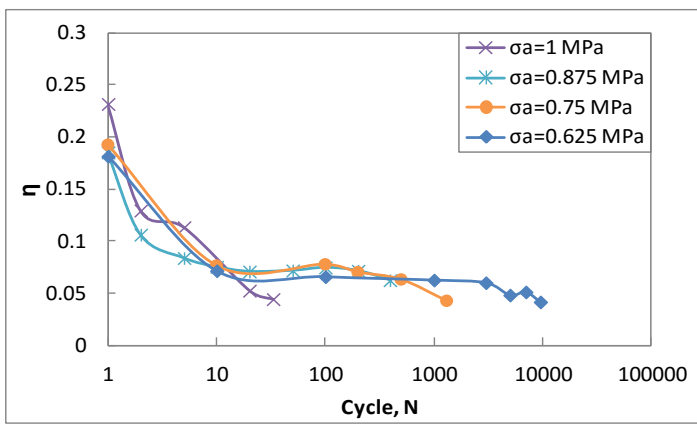

(d) $30 \%$

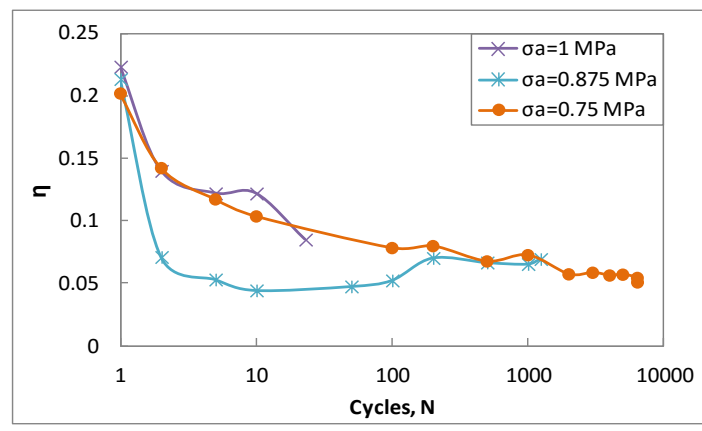

(e) $35 \%$

Figure 9 Damping loss factor versus cycles for isotropic MREs with different CI content, (a) $15 \%$; (b) $20 \%$; (c) $25 \%$; (d) $30 \%$; (e) $35 \%$. Samples were dynamically tested in the absence of a magnetic field

Table 5 Values of $\eta$ at failure for MREs with different CI content

\begin{tabular}{ccc}
\hline $\begin{array}{c}\text { CI volume } \\
\text { content }(\%)\end{array}$ & $\boldsymbol{\eta}$ (with magnetic fields) & $\begin{array}{c}\boldsymbol{\eta} \text { (without magnetic } \\
\text { fields) }\end{array}$ \\
\hline 15 & $0.025-0.043$ & $0.019-0.045$ \\
20 & $0.030-0.048$ & $0.019-0.046$ \\
25 & $0.036-0.056$ & $0.033-0.051$ \\
30 & $0.042-0.062$ & $0.042-0.062$ \\
35 & $0.058-0.080$ & $0.045-0.079$ \\
\hline
\end{tabular}

\section{Conclusions}

Equi-biaxial fatigue behaviour of silicone based MREs in the presence of external magnetic fields was investigated. It was found that for the same carbonyl iron content and testing conditions, the fatigue life of a silicone based MRE in a magnetic field was 
higher than that without a magnetic field. Magnetic fields have a great influence on the stress-strain behaviour of MREs. Achieving large deformations in MRE samples took longer when they were tested in magnetic fields. The decrease in the value of $\mathrm{E}^{*}$ was more gradual in the presence of magnetic fields and this was particularly evident at lower stress amplitudes. A limiting value of $\mathrm{E}^{*}$ at failure was reached for each material and the values of $E^{*}$ at failure were within a quite small range when the samples were tested both with and without a magnetic field. As previously found (Zhou et al., 2015), dynamic stored energy can also be used as a plausible predictor in determining the fatigue life of MREs when they are subjected to external magnetic fields. However, the magnetic field did not have a significant influence on the damping loss factor for the range of MREs tested. Nonetheless, the experimentation showed that the damping properties of MREs can be maintained at a stable level during their entire service lives, after conditioning has taken place. Together with previous findings in respect of MRE properties in the absence of magnetic fields, the parameters obtained relating fatigue life to stress amplitudes and dynamic stored energy in this research provide a useful reference for the development of constitutive models for characterising the complex dynamic behaviour of MREs.

\section{Acknowledgements}

The authors would like to give thanks for the financial support from the Irish Research Council (previously the Irish Research Council for Science, Engineering and Technology, IRCSET) and the use of facilities in the Focas Research Institute, Dublin Institute of Technology.

\section{References}

Alshuth T, Abraham F and Jerrams S. (2002) Parameter Dependence and Prediction of Fatigue Properties of Elastomer Products. Rubber Chemistry and Technology 75: 635-642.

Ayoub G, Naït-Abdelaziz M, Zaïri F, et al. (2012) Fatigue life prediction of rubber-like materials under multiaxial loading using a continuum damage mechanics approach: Effects of two-blocks loading and R ratio. Mechanics of Materials 52: 87-102.

Böse H and Röder R. (2009) Magnetorheological elastomers with high variability of their mechanical properties. Journal of Physics: Conference Series. IOP Publishing, 012090.

Bellan C and Bossis G. (2002) Field dependence of viscoelastic properties of MR elastomers. International Journal of Modern Physics B 16: 2447-2453.

Boczkowska A and Awietjan S. (2012) Microstructure and Properties of Magnetorheological Elastomers. Advanced Elastomers-Technology, Properties and Applications.

Boczkowska A and Awietjan SF. (2009) Urethane magnetorheological elastomers-manufacturing, microstructure and properties. Solid State Phenomena. Trans Tech Publ, 107-112.

Boczkowska A, Awietjan SF and Wroblewski R. (2007) Microstructure-property relationships of urethane magnetorheological elastomers. Smart Materials and Structures 16: 1924.

Cadwell SM, Merrill RA, Sloman CM, et al. (1940) Dynamic Fatigue Life of Rubber. Industrial \& Engineering Chemistry Analytical Edition 12: 19-23. 
Elie LD, Ginder JM, Nichols ME, et al. (1998) Method and apparatus for reducing brake shudder. Google Patents.

Farshad M and Le Roux M. (2005) Compression properties of magnetostrictive polymer composite gels. Polymer Testing 24: 163-168.

Fuchs A, Gordaninejad F and Hitchcock GH. (2006) Controllable magneto-rheological elastomer vibration isolator. Google Patents.

Gong XL, Zhang XZ and Zhang PQ. (2005) Fabrication and characterization of isotropic magnetorheological elastomers. Polymer Testing 24: 669-676.

Hoang N, Zhang N and Du H. (2009) A dynamic absorber with a soft magnetorheological elastomer for powertrain vibration suppression. Smart Materials and Structures 18: 074009.

Ju BX, Tang R, Zhang DY, et al. (2015) Temperature-dependent dynamic mechanical properties of magnetorheological elastomers under magnetic field. Journal of Magnetism and Magnetic Materials 374: 283-288.

Lokander M, Reitberger T and Stenberg B. (2004) Oxidation of natural rubber-based magnetorheological elastomers. Polymer Degradation and Stability 86: 467-471.

Lokander M and Stenberg B. (2003) Improving the magnetorheological effect in isotropic magnetorheological rubber materials. Polymer Testing 22: 677-680.

Mars WV. (2002) Cracking Energy Density as a Predictor of Fatigue Life under Multiaxial Conditions. Rubber Chemistry and Technology 75: 1-17.

Mars WV and Fatemi A. (2002) A literature survey on fatigue analysis approaches for rubber. International Journal of Fatigue 24: 949-961.

Murphy N. (2010) Providing Stress Controlled Equi-Biaxial Fatigue Test Data for Elastomers using the Bubble Inflation Method. Dublin: Dublin Institute of Technology.

Rivlin RS and Thomas AG. (1953) Rupture of rubber. I. Characteristic energy for tearing. Journal of Polymer Science 10: 291-318.

Roylance D. (2001) Stress-strain curves. Massachusetts Institute of Technology study, Cambridge.

Schubert G and Harrison P. (2015) Large-strain behaviour of Magneto-Rheological Elastomers tested under uniaxial compression and tension, and pure shear deformations. Polymer Testing 42: 122-134.

Watson JR. (1997) Method and apparatus for varying the stiffness of a suspension bushing. Google Patents.

Yim JH, Cho SY, Seo YJ, et al. (2003) A study on material damping of $0^{\circ}$ laminated composite sandwich cantilever beams with a viscoelastic layer. Composite Structures 60: 367-374.

Zhang W, Gong X, Jiang W, et al. (2010) Investigation of the durability of anisotropic magnetorheological elastomers based on mixed rubber. Smart Materials and Structures 19: 085008.

Zhou Y, Jerrams S, Betts A, et al. (2014) Fatigue life prediction of magnetorheological elastomers subjected to dynamic equi-biaxial cyclic loading. Materials Chemistry and Physics 146: 487-492.

Zhou Y, Jerrams S, Betts A, et al. (2015) The influence of particle content on the equi-biaxial fatigue behaviour of magnetorheological elastomers. Materials \& Design 67: 398-404. 